\title{
THE LOOP-SPACE FUNCTOR IN HOMOLOGICAL ALGEBRA
}

\author{
BY \\ ALEX HELLER( ${ }^{(1)}$
}

This note constitutes a sequel to $[\mathrm{AC}]$, the terminology and notation of which are used throughout. Its purpose is to contribute some technical devices, viz. the notion of an ideal, and that of the loop-space functor, to the study of homological algebra.

The concept of an ideal of an additive category is introduced (\$1) in analogy with the familiar notion of the theory of rings. In particular the quotient of a category by a two-sided ideal is defined. Of especial interest are the ideals generated by identity maps, which are studied in $\$ 2$.

For an abelian category with enough projectives a functor $\Omega$, defined on the quotient of the category by its projectives ( $\$ 3)$, bears a close analogy to the loop-space functor of topology. This functor, as an operation on modules, has also been considered by P. J. Hilton and B. Eckmann [H-E].

If $\mathcal{K}$ is an abelian category with enough projectives then for $\mathcal{K}^{s}$, the category of proper s.e.s. in $\mathcal{K}$, a functor $\Gamma$ is introduced ( $\$ 4$ ). Pursuing the topological analogy, $\Gamma$ plays the role of the operation which turns inclusion maps into fibre maps. This is related to the loop-space functor by the operation (\$5) that its threefold iterate is just $\Omega$ for the category $\kappa^{s}$ (a fact which is known for the topological analogue). Using this result it is possible to associate to a proper s.e.s. exact sequences of homomorphism groups in the original category modulo its projectives.

Finally the Ext groups of the original category are related to the homomorphism groups in the quotient by projectives ( $\$ 6)$ by a functor which exhibits the latter as quotient groups of the former. The kernel measures the extent to which projectives fail to be injective; if they are all injective the study of Ext is reduced to that of the quotient category and the functor $\Omega$.

As in $[\mathrm{AC}]$ the theory is subject to straightforward dualization which however is barely indicated.

The general reference is $[A C]$. To this should be added [G] which had not yet appeared when $[\mathrm{AC}]$ was written. It should be noted that "additive category" in $[G]$ is used in a sense different from the present one, and that "abelian category" in [G] is equivalent to the "exact category" of Buchsbaum and $[\mathrm{AC}]$.

1. Ideals in an additive category. A left ideal $\subseteq$ of an additive category $\mathcal{K}$ is a subset of the maps of $\mathcal{K}$ with the properties:

Received by the editors October 26, 1959.

(1) Fellow of the Alfred P. Sloan Foundation. 
1.1. for each $A, B \in \mathscr{K}$ the intersection $\subseteq \cap \operatorname{Hom}(A, B)$ is a subgroup of $\operatorname{Hom}(A, B)$;

I.2. if $f: A \rightarrow B, g: B \rightarrow C$ and $f \in \subseteq$ then $g f \in \subseteq$.

Right ideals and two-sided ideals are defined by the obvious alterations of I.2. Only two-sided ideals will be considered below; they will be referred to simply as ideals.

It is easy to see that the intersection of any set of ideals is an ideal; also if $\left\{\mathfrak{S}_{i}\right\}$ is a collection of ideals then $\sum_{i} \mathfrak{S}_{i}$, defined by $\left(\sum_{i} \mathfrak{S}_{i}\right) \cap \operatorname{Hom}(A, B)$ $=\sum_{i}\left(\mathfrak{S}_{i} \cap \operatorname{Hom}(A, B)\right)$, is an ideal.

An object $A \in \mathcal{K}$ is said to be in an ideal $\subseteq$ if its identity map 1:A is in 5. If $B$ is a direct factor of $A$, i.e. if $f: A \rightarrow B, g: B \rightarrow A, g f=1: B$, and $A$ is in $\subseteq$ then so is $B$. In particular if $B$ is equivalent to $A$ then both or neither are in an ideal. A direct sum, whether of objects or of maps, is in an ideal if and only if both summands are.

If $F: \mathfrak{K} \rightarrow \mathfrak{L}$ is an additive functor and $\mathfrak{T}$ is an ideal in $\mathscr{L}$ then $F^{-1}(\mathfrak{T})$ is an ideal in $\mathcal{K}$. If in particular $\mathfrak{T}$ is the ideal consisting of all the 0 -maps in $\mathfrak{L}$ then $F^{-1}(\mathfrak{T})$ is the kernel of $F$.

If $\mathfrak{S}$ is an ideal in $\mathscr{K}$ the quotient category $\mathscr{K} / \mathfrak{S}$ is defined as follows: $\Re / \subseteq$ has the same objects as $\mathscr{K}$, while for any $A, B$,

$$
\operatorname{Hom}(A, B ; \Re / S)=\operatorname{Hom}(A, B ; \mathfrak{K}) / \subseteq \cap \operatorname{Hom}(A, B ; \Re) \text {; }
$$

composition in $\mathcal{K} / \mathfrak{S}$ is defined by composition in $\mathcal{K}$, in such a way as to make the canonical map $\pi: \mathfrak{K} \rightarrow \mathscr{K} / \subseteq$ a covariant functor. The kernel of $\pi$ is clearly $\mathfrak{S}$, so that every ideal is the kernel of a functor.

If $\mathfrak{S}, \mathfrak{I}$ are ideals in $\mathfrak{K}, \mathfrak{L}$ and $F: \mathfrak{K} \rightarrow \mathfrak{L}$ takes $\mathfrak{S}$ into $\mathfrak{I}$ then a unique functor $\bar{F}: \mathscr{K} / \mathfrak{S} \rightarrow \mathscr{L} / \mathfrak{T}$ is defined by commutativity in

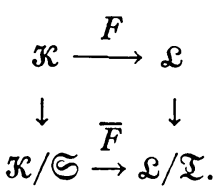

In particular if $\subseteq$ is contained in the kernel of $F$ then $\bar{F}: \nVdash / \subseteq \rightarrow \mathcal{L}$.

It should be noticed here that, in contradistinction to the first isomorphism theorem for rings, even if $F$ is (in any sense) onto, $\bar{F}: \mathfrak{K} /($ kernel $F) \rightarrow \mathscr{L}$ need not be an isomorphism.

The notion of an invariant may conveniently be expressed in terms of ideals. If $\mathfrak{S}$ is an ideal of $\mathcal{K}$ an invariant of $\mathcal{K}$ with respect to $\subseteq$ is a functor which vanishes on $\mathfrak{S}$, or, equivalently, a functor on $\nVdash / \mathfrak{S}$. For example, the set of nullhomotopic maps in $d^{p} \mathcal{K}$, the category of objects-with-properderivation in $\mathcal{K}$, is a two-sided ideal $\mathfrak{N}\left[d^{p} \mathcal{K}\right]$. The homology functor $H: d^{p} \mathfrak{K} \rightarrow \mathcal{K}$ is an invariant with respect to $\mathfrak{N}\left[d^{p} \mathfrak{K}\right]$, or a homotopy invariant.

2. Object-ideals. If $\mathcal{K}$ is an additive category any set of maps in $\mathcal{K}$ is contained in a smallest ideal; viz. the intersection of all the ideals containing 
it. This ideal may be said to be generated by the set of maps. In particular, for any set of objects in $\nVdash$, the set of their identity maps generates an ideal which is then said to be generated by the set of objects.

A set $\mathfrak{M}$ of objects in an additive category $\mathscr{K}$ will be called additive if (1) whenever $A \in \mathfrak{M}$ and $B$ is a direct factor of $A$ then $B \in \mathfrak{M}$, (2) a direct sum of two objects of $\mathfrak{M}$ is in $\mathfrak{M}$. If $\nVdash$ has cancellation, (1) and (2) may be replaced by the condition that a direct sum is in $\mathfrak{M}$ if and only if both summands are. Clearly any set of objects in $\nVdash$ is contained in a smallest additive set, consisting of all direct factors of direct sums of elements of the set.

Proposition 2.1. Let $\mathfrak{M}$ be a set of objects in $\mathfrak{K}$, which generates an ideal $\mathfrak{S}$. Then if $\mathfrak{M}^{+}$is the smallest additive set containing $\mathfrak{M}$,

(a) a map $f: A \rightarrow B$ is in $S$ if and only if it can be factored through an element of $\mathfrak{M}^{+}$, i.e. if there is a $C \in \mathfrak{M}^{+}, g: A \rightarrow C, h: C \rightarrow B$ such that $h g=f$;

(b) the set of objects in $\subseteq$ is just $\mathfrak{M}^{+}$.

Observe first that $\mathfrak{M}^{+}$is certainly contained in $\subseteq$, thus also the maps described in (a). But this set is an ideal containing $\mathfrak{M}$, hence must be $\mathfrak{S}$. The remaining condition follows on applying (a) to the identity map of an object in $\mathfrak{S}$.

If $\mathfrak{S}$ is an ideal in $\mathscr{K}$ and $\pi: \mathscr{K} \rightarrow \mathscr{K} / \mathfrak{S}$ is the canonical functor it may happen for a map $f \in \varkappa$ that $\pi f$ is an equivalence, even though $f$ itself is not. If the ideal is of the type just discussed and $\mathscr{K}$ has cancellation a precise account may be given of the circumstances in which this phenomenon occurs.

THEOREM 2.2. Let $\mathfrak{M}$ be an additive set of objects in a category $\mathcal{K}$ with cancellation, generating an ideal $\mathfrak{S}$, and let $\pi: \mathcal{K} \rightarrow \mathcal{K} / \mathfrak{S}$ be the canonical functor. Suppose $f: A \rightarrow B$ in $\Re$. Then $\pi f$ is an equivalence if and only if there are objects $A^{\prime}, B^{\prime} \in \mathfrak{M}$ and an equivalence $\bar{f}: A+A^{\prime} \rightarrow B+B^{\prime}$ in $\Re$ such that

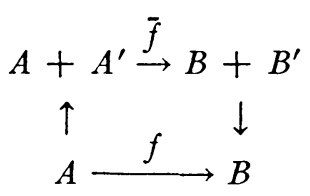

commutes in $\mathcal{K}$, the vertical arrows being, respectively, the injection and the projection of the indicated direct sum decompositions.

In particular, $A, B \in \Re$ are equivalent in $\Re / \subseteq$ if and only if, for some $A^{\prime}, B^{\prime} \in \mathfrak{M}, A+A^{\prime}$ and $B+B^{\prime}$ are equivalent in $\mathcal{K}$.

To see the sufficiency of the condition, let $\left(A, A^{\prime}, i, j, i^{\prime}, j^{\prime}\right)$ be the direct sum decomposition of $A+A^{\prime}$. Then $j i=1: A$ while $i j=1:\left(A+A^{\prime}\right)-i^{\prime} j^{\prime}$ whence $\pi(i j)=(\pi i)(\pi j)=1:\left(A+A^{\prime}\right)$ in $\mathscr{K} / \mathfrak{S}$. The injection and projection are thus equivalences in $\Re / S$.

To prove the necessity, suppose $\pi f$ is an equivalence in $\mathscr{K} / \subseteq$. Then it has an inverse $\pi g$, and $g$ satisfies $g f-1: A \in \subseteq, f g-1: B \in \subseteq$. By 2.1 , then, 
gf-1: $A=t s$ where $s: A \rightarrow B^{\prime}, t: B^{\prime} \rightarrow A, B^{\prime} \in \mathfrak{M}$. Let $B+B^{\prime}$ be a direct sum of $B$ and $B^{\prime}$ with decomposition $\left(B, B^{\prime}, i, j, i^{\prime}, j^{\prime}\right)$. Then if $+i^{\prime} s: A \rightarrow B+B^{\prime}$ and $g j+t j^{\prime}: B+B^{\prime} \rightarrow A$. But $\left(g j+t j^{\prime}\right)\left(i f+i^{\prime} s\right)=g f+t s=1: A$.

Since $\mathcal{K}$ has cancellation there is a direct sum decomposition $\left(A, A^{\prime}, i f+i^{\prime} s, g j+t j^{\prime}, k, m\right)$ of $B+B^{\prime}$. Since $f=j\left(i f+i^{\prime} s\right)$ it remains only to show that $A^{\prime} \in \mathfrak{M}$. But $i j+i^{\prime} j^{\prime}=\left(i f+i^{\prime} s\right)\left(g j+t j^{\prime}\right)+k m \equiv i f g j+k m$ $\equiv i j+k m \bmod \subseteq$. Thus $k m \equiv i^{\prime} j^{\prime} \equiv 0 \bmod \subseteq$. Finally, $1: A^{\prime}=m k=m(k m) k$ $\equiv 0 \bmod \subseteq$, which completes the proof.

A theorem on homotopy type, due originally to J. H. C. Whitehead, constitutes an application. If $\mathcal{K}$ is an abelian category the ideal $\mathfrak{N}[d \Re]$ of nullhomotopic maps in the category of objects-with-derivation, is generated by the set of null-objects $A^{x}, A \in \mathcal{K}$.

ThEOREM 2.3. If $\mathcal{K}$ is an abelian category, objects $A, B$ of $d \mathfrak{K}$ are of the same homotopy type if and only if there are objects $A_{0}, B_{0}$ of $\mathfrak{K}$ such that $A+A_{0}^{x}$ and $B+B_{0}^{x}$ are equivalent in $d \mathscr{K}$.

3. The loop-space functor. Suppose that $\mathscr{K}$ is an abelian category with enough projectives and denote by $\mathfrak{B}[\mathfrak{K}]$ the ideal generated by the projectives. Since the projectives form an additive set, the maps of $\mathfrak{B}[\mathcal{K}]$ are exactly those which can be factored through some projective; it is easy to see that they have the following property:

Lemma 3.1. If $\phi: B \rightarrow B^{\prime \prime}$ is a proper epimorphism and $f: A \rightarrow B^{\prime \prime}$ is in $\mathfrak{P}[\mathcal{K}]$ then there is an $\bar{f}: A \rightarrow B$ in $\mathfrak{P}[\mathcal{K}]$ such that $\phi \bar{f}=f$.

Suppose further that an assignment of projectives has been made in $\mathcal{K}$, i.e. that to every $A \in \mathcal{K}$ has been associated a proper s.e.s.

$$
P A=(0 \rightarrow \Omega A \stackrel{\omega A}{\rightarrow} P A \stackrel{p A}{\rightarrow} A \rightarrow 0)
$$

with $P A$ projective. Then if

$$
B=\left(0 \rightarrow B^{\prime} \stackrel{b^{\prime}}{\rightarrow} \rightarrow B \stackrel{b^{\prime \prime}}{\rightarrow} \rightarrow B^{\prime \prime} \rightarrow 0\right)
$$

is any proper s.e.s. and $f^{\prime \prime}: A \rightarrow B^{\prime \prime}$ any map there is a map $f: P A \rightarrow B$ such that $b^{\prime \prime} f=f^{\prime \prime}(p A)$, and hence a map $\left(f^{\prime}, f, f^{\prime \prime}\right): P A \rightarrow B$. If also $\left(\bar{f}^{\prime}, \bar{f}, f^{\prime \prime}\right): P A \rightarrow B$ then $\left(\bar{f}^{\prime}-f^{\prime}, \bar{f}-f, 0\right): P A \rightarrow B$ and thus $\bar{f}^{\prime}-f^{\prime}=g(\omega A)$ for $g: P A \rightarrow B^{\prime}$, so that $\bar{f}^{\prime}-f^{\prime} \in \mathfrak{B}[\mathcal{K}]$. In other words the pair $B, f^{\prime \prime}$ determines by this construction a unique element of

$$
\operatorname{Hom}\left(\Omega A, B^{\prime} ; \mathfrak{K} / \mathfrak{P}[\mathfrak{K}]\right) \text {. }
$$

It is enough to consider two special cases of this construction: applied to sequences $P A, P B$, for $A, B \in \mathcal{K}$, and maps $f: A \rightarrow B$, it gives a covariant additive functor $\mathfrak{K} \rightarrow \mathcal{K} / \mathfrak{B}[\mathcal{K}]$ which, since it vanishes on $\mathfrak{P}[\mathcal{K}]$, is just the composition of the identification functor $\pi: \mathcal{K} \rightarrow \mathcal{K} / \mathfrak{B}[\mathcal{K}]$ with a functor $\Omega: \mathfrak{K} / \mathfrak{B}[\mathcal{K}] \rightarrow \mathfrak{K} / \mathfrak{B}[\mathcal{K}]$. This is the loop-space functor of $\mathcal{K}$. It depends of 
course on the assignment of projectives, but if two of these are considered the same construction yields a natural equivalence of the two loop-space functors.

Applied on the other hand to proper s.e.s. $A=\left(0 \rightarrow A^{\prime} \rightarrow A \rightarrow A^{\prime \prime} \rightarrow 0\right)$, and to the identity map of $A^{\prime \prime}$, it gives a natural transformation $\sigma: \pi S^{\prime} \rightarrow \Omega \pi S^{\prime \prime}$ of the functors $\pi S^{\prime}, \Omega \pi S^{\prime \prime}: K^{s} \rightarrow \mathcal{K} / \mathfrak{B}[\mathcal{K}]$. The general case, i.e. that first discussed above yields (adopting the original notation) the map $f^{\prime}=(\sigma B)\left(\Omega f^{\prime \prime}\right)$ $\in \operatorname{Hom}\left(\Omega A, B^{\prime} ; \Re / \mathfrak{P}[\Re]\right)$.

The justification for the name "loop-space," first adopted by Hilton and Eckmann, is in part analogy: for "epimorphism" read "fibre map," for "projective" read "contractible," and for "kernel" read "fibre." There is however at least one case in which the loop-space functor here defined is nothing more or less than the classical loop-space functor.

The category of abelian group-complexes is an exact category, "exact sequences" having the naive meaning. An epimorphism is always a fibre map, in the sense of semi-simplicial complexes, and its kernel is the fibre. It remains only to observe that there are enough projectives, and that a connected projective is contractible.

The notion of projective dimension is conveniently expressible in terms of the loop-space functor: the projective dimension of an object $A \in \mathcal{K}$ is inf $\left\{n \mid \Omega^{n+1} A=0\right\}$; in the same spirit, the projective dimension of a map $f: A \rightarrow B$ is inf $\left\{n \mid \Omega^{n+1} f=0\right\}$, which is of course no larger than the minimum of the dimensions of $A$ and $B$. The projective dimension of the category is just the supremum of the projective dimensions of its objects.

If a category $\nVdash$ has enough injectives, which generate the ideal $\mathfrak{B}^{*}[\mathcal{K}]$, the dual functor $\Omega^{*}: \mathfrak{K} / \mathfrak{B} *[\mathfrak{K}] \rightarrow \mathscr{K} / \mathfrak{B}^{*}[\mathcal{K}]$ is defined. This is known as the suspension functor. Also, the natural transformation $\sigma^{*}: \pi^{*} S^{\prime} \rightarrow \Omega^{*} \pi^{*} S^{\prime \prime}$ dual to $\sigma$, is defined. The injective dimension of an object, map or category is of course defined in terms of the functor $\Omega^{*}$.

An important special case is that of a Frobenius category, i.e. a category which has both enough projectives and enough injectives, and in which these two classes coincide, so that $\mathfrak{P}[\Re]=\mathfrak{B}^{*}[\mathfrak{K}]$. The obvious example is the one which suggests the name: the category of finite dimensional left modules of a Frobenius algebra.

It is clear that in a Frobenius category $K$ the compositions $\Omega^{*} \Omega$ and $\Omega \Omega^{*}$ are both naturally equivalent to the identity functor of $\Re / \mathfrak{P}[\Re]$. In fact it does no great violence to assume that $\Omega$ and $\Omega^{*}$ are inverse.

Lemma 3.2. If $\mathcal{K}$ is a Frobenius category and $B$ is equivalent to $\Omega A$ in $\Re / \mathfrak{B}[\mathcal{K}]$ then there is a proper s.e.s. $0 \rightarrow B \rightarrow X \rightarrow A \rightarrow 0$ with $X$ projective.

For $B+B^{\prime}$ is equivalent in $\mathscr{K}$ to $\Omega A+A^{\prime}$ for some $A^{\prime}, B^{\prime} \in \mathfrak{B}[\mathfrak{K}]$. Thus there is a proper s.e.s. $0 \rightarrow B+B^{\prime} \rightarrow P A+A^{\prime} \rightarrow A \rightarrow 0$ from which the commutative diagram 


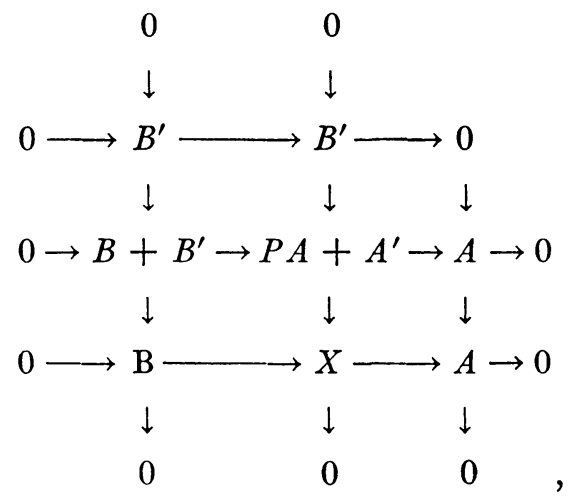

having proper exact rows and columns, may be constructed. Since $B^{\prime}$ is injective as well as projective the center column splits and $X$ is also projective.

Now suppose $\mathfrak{A}$ is an equivalence class of objects in $\mathscr{K} / \mathfrak{P}[\mathcal{K}]$. Then for any $A \in \mathfrak{A}$, and any assignment of projectives, $\Omega A$ is in an equivalence class $\Omega \mathfrak{A}$ depending only on $\mathfrak{A}$. If for each $\mathfrak{A}$, these two classes have the same cardinal number, then, clearly, assignments of injectives and projectives in $\Re$ may be made in such a way that $\Omega^{*} \Omega=\Omega \Omega^{*}$ is the identity on $\mathscr{K} / \mathfrak{P}[\mathcal{K}]$.

This does not seem a particularly restrictive hypothesis on $\mathcal{K}$; in an obvious way it is always possible to adjoin new objects, equivalent to old ones, wherever they are needed, without essentially changing the structure of the category. However, the condition seems rather more metamathematical than the others considered, and will not be incorporated into the definition of a Frobenius category.

In any case the following statement is obviously true.

THEOREM 3.3. In a Frobenius category the projective (injective) dimension of any object or map is either 0 or $\infty$.

4. Loop-space and permutation in $\kappa^{s}$. If $\mathfrak{K}$ is an abelian category with enough projectives then so is $\mathcal{K}^{s}$, the category of proper s.e.s. in $\mathcal{K}$, and in fact $[\mathrm{AC}]$ the projectives of $\varkappa^{s}$ are just the s.e.s. $0 \rightarrow X^{\prime} \rightarrow X \rightarrow X^{\prime \prime} \rightarrow 0$ with $X^{\prime}, X^{\prime \prime}$ projective in $\Re$. Thus $S^{\prime}, S, S^{\prime \prime}: \Re^{s} \rightarrow \mathcal{K}$ all take $\mathfrak{B}\left[\mathfrak{K}^{s}\right]$ into $\mathfrak{B}[\Re]$. The associated functors $\mathfrak{K}^{\mathfrak{s}} / \mathfrak{P}\left[\mathfrak{K}^{\mathfrak{s}}\right] \rightarrow \mathfrak{K} / \mathfrak{P}[\mathfrak{K}]$ will be denoted by the same symbols.

If $A=\left(0 \rightarrow A^{\prime} \rightarrow a^{\prime} \rightarrow A \rightarrow a^{a^{\prime \prime}} \rightarrow A^{\prime \prime} \rightarrow 0\right)$ is a proper s.e.s. in $\mathcal{K}$ then $S^{\prime} P A$, $S P A, S^{\prime \prime} P A$ are proper s.e.s. in $\mathcal{K}$ terminating in $A^{\prime}, A, A^{\prime \prime}$. Thus there are natural equivalences $S^{\prime} \Omega \rightarrow \Omega S^{\prime}, S \Omega \rightarrow \Omega S, S^{\prime \prime} \Omega \rightarrow \Omega S^{\prime \prime}$ of the composite functors $K^{s} / \mathfrak{P}\left[K^{s}\right] \rightarrow \mathscr{K} / \mathfrak{B}[K]$ which have of course the property that

$$
\begin{aligned}
& S^{\prime} \Omega A \stackrel{s^{\prime} \Omega A}{\longrightarrow} S \Omega A \stackrel{s^{\prime \prime} \Omega A}{\longrightarrow} S^{\prime \prime} \Omega A
\end{aligned}
$$

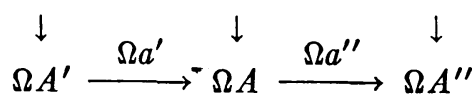


commutes. These will be called the canonical commutation equivalences.

In $\mathcal{K}^{s} / \mathfrak{P}\left[\mathcal{K}^{s}\right]$ another operation may be introduced, which plays, in relation to the loop-space functor, the same role which in the theory of fibre spaces is played by the functor which turns inclusion maps into fibre maps. If $A \in K^{s}$, as above, then $s^{\prime \prime} \Gamma A: A^{\prime}+P A \rightarrow A$, the "sum" of $a^{\prime}$ and $p A$, is a proper epimorphism, and occurs in a proper s.e.s.

$$
\Gamma A=\left(0 \rightarrow S^{\prime} \Gamma A \stackrel{s^{\prime} \Gamma A}{\longrightarrow} A^{\prime}+P A \stackrel{s^{\prime \prime} \Gamma A}{\longrightarrow} A \rightarrow 0\right) .
$$

If $f=\left(f^{\prime}, f, f^{\prime \prime}\right): A \rightarrow B$ in $\mathcal{K}^{s}$ then there is a $g: P A \rightarrow P B$ such that $(p B) g$ $=f(p A)$ so that $\left(s^{\prime \prime} \Gamma B\right)\left(f^{\prime}+g\right)=f\left(s^{\prime \prime} \Gamma A\right)$, giving a map $h: \Gamma A \rightarrow \Gamma B$. If $g^{\prime}$ satisfies the same condition, giving rise to $h^{\prime}: \Gamma A \rightarrow \Gamma B$ then $h^{\prime}-h$ can be factored through $0 \rightarrow P A \rightarrow P A \rightarrow 0 \rightarrow 0$ and is thus in $\mathfrak{B}\left[\mathfrak{K}^{s}\right]$. Similarly, if $f \in \mathfrak{B}\left[\mathcal{K}^{s}\right]$ then $h \in \mathfrak{B}\left[\mathcal{K}^{s}\right]$. If $\Gamma f$ denotes the class of $h$ then $\Gamma: \mathcal{K}^{s} / \mathfrak{B}\left[\mathcal{K}^{s}\right]$ $\rightarrow K^{s} / \mathfrak{B}\left[\mathfrak{K}^{s}\right]$; this will be referred to as the permutation functor of $\mathfrak{K}^{s}$. It is easy to see that a different assignment of projectives gives a naturally equivalent functor.

Now suppose that $A$ is a proper s.e.s. in $\Re$, that $\left(A^{\prime}, P A, i, j, i^{\prime}, j^{\prime}\right)$ is a direct sum decomposition of $A^{\prime}+P A$, and consider the commutative diagram

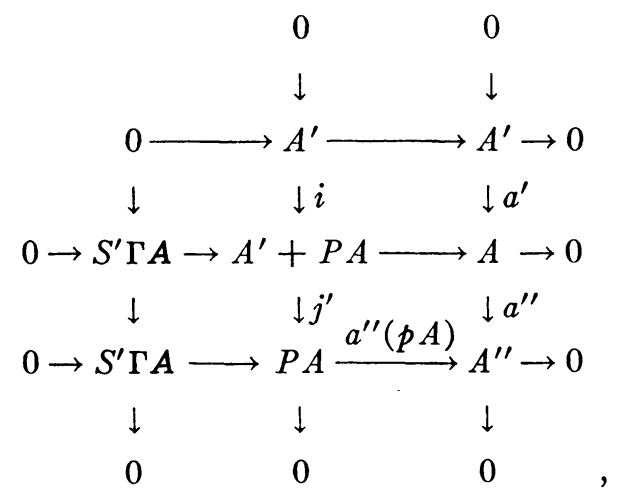

in which the second row is $\Gamma A$. If $C$ denotes the last row then $\sigma C: \Omega A^{\prime \prime} \rightarrow S^{\prime} \Gamma A$ is an equivalence in $\mathscr{K} / \mathfrak{B}[\mathcal{K}]$. This is the inverse of a natural equivalence $S^{\prime} \Gamma \rightarrow \Omega S^{\prime \prime}$ which together with the projection $S \Gamma A \rightarrow S^{\prime} A$ and the identity $S^{\prime \prime} \Gamma A=S A$, constitute the canonical permutation equivalences.

Proposition 4.3. The diagrams

$$
\begin{aligned}
& S^{\prime} \Gamma A \stackrel{s^{\prime} \Gamma A}{\longrightarrow} S \Gamma A \stackrel{s^{\prime \prime} \Gamma A}{\longrightarrow} S^{\prime \prime} \Gamma A \\
& \begin{array}{cccr}
\downarrow & * & \downarrow & \\
\Omega S^{\prime \prime} A \stackrel{\sigma A}{\longrightarrow} S^{\prime} A \stackrel{S^{\prime} A}{\longrightarrow} S A
\end{array}
\end{aligned}
$$

in $\mathfrak{K} / \mathfrak{B}[\mathfrak{K}]$, in which the vertical arrows represent the permutation equivalences, commute in the unstarred squares and anticommute in the starred squares. 
Only the anticommutativity needs proof. There is a map $\left(\beta^{\prime}, \beta, 1: A^{\prime \prime}\right): C$ $\rightarrow P A^{\prime \prime}$, since $P A$ is projective, and $\beta^{\prime}$ represents $(\sigma C)^{-1}$, hence the permutation equivalence. If also $\left(\alpha^{\prime}, \alpha, 1: A^{\prime \prime}\right): P A^{\prime \prime} \rightarrow A$, so that $\alpha^{\prime}$ represents $\sigma A$ then $\left(\alpha^{\prime} \beta^{\prime}, \alpha \beta, 1: A^{\prime \prime}\right): C \rightarrow A$ and $\alpha^{\prime} \beta^{\prime}$ represents one of the compositions to be compared, the other being $j\left(s^{\prime} \Gamma A\right)$. There is however a map $\left(\gamma, p A, 1: A^{\prime \prime}\right): C$ $\rightarrow A$, so that $\gamma \equiv \alpha^{\prime} \beta^{\prime} \bmod \mathfrak{B}[\mathfrak{K}]$.

Now

$$
\begin{aligned}
a^{\prime}\left(\gamma+j\left(s^{\prime} \Gamma A\right)\right) & =(p A) j^{\prime}\left(s^{\prime} \Gamma A\right)+a^{\prime} j\left(s^{\prime} \Gamma A\right) \\
& =\left[(p A) j^{\prime}+a^{\prime} j\right]\left(s^{\prime} \Gamma A\right)\left(s^{\prime \prime} \Gamma A\right)\left(s^{\prime} \Gamma A\right)=0,
\end{aligned}
$$

which completes the proof.

In the presence of enough injectives a dual permutation, which need not be explicitly introduced, may be constructed.

5. The permutation functor of $\varkappa^{s}$, continued. The functor $\Gamma$ introduced in $\S 4$ has the following remarkable property.

THEOREM 5.1. If $K$ is an abelian category with enough projectives then the functors $\Gamma^{3}, \Omega: K^{s} / \mathfrak{P}\left[\mathfrak{K}^{s}\right] \rightarrow K^{s} / \mathfrak{P}\left[\mathfrak{K}^{s}\right]$ are naturally equivalent.

Adopting once more the notation of $\$ 4$, introduce the direct sum decompositions

$$
\begin{aligned}
& \left(S^{\prime} \Gamma A, P S \Gamma A, i_{1}, j_{1}, i_{1}^{\prime}, j_{1}^{\prime}\right) \text { of } S^{\prime} \Gamma A+P S \Gamma A=S \Gamma^{2} A=S^{\prime \prime} \Gamma^{3} A, \\
& \left(S^{\prime} \Gamma^{2} A, P S \Gamma^{2} A, i_{2}, j_{2}, j_{2}^{\prime}, j_{2}^{\prime}\right) \text { of } S^{\prime} \Gamma^{2} A+P S \Gamma^{2} A=S \Gamma^{3} A
\end{aligned}
$$

so that $s^{\prime \prime} \Gamma^{2} A=\left(s^{\prime} \Gamma A\right) j_{1}+(p S \Gamma A) j_{1}^{\prime}$ and $s^{\prime \prime} \Gamma^{3} A=\left(s^{\prime} \Gamma^{2} A\right) j_{2}+\left(p S \Gamma^{2} A\right) j_{2}^{\prime}$, as well as the direct sum decompositions

$$
\begin{aligned}
& \left(P S A, P S \Gamma A, u_{1}, v_{1}, u_{1}^{\prime}, v_{1}^{\prime}\right) \text { of } P S A+P S \Gamma A, \\
& \left(P S \Gamma A, P S \Gamma^{2} A, u_{2}, v_{2}, u_{2}^{\prime}, v_{2}^{\prime}\right) \text { of } P S \Gamma A+P S \Gamma^{2} A,
\end{aligned}
$$

and consider the diagram

$$
\begin{gathered}
0 \longrightarrow S^{\prime} \Gamma^{2} A+P S \Gamma A \stackrel{\phi}{\longrightarrow} P S \Gamma A+P S \Gamma^{2} A \stackrel{\psi}{\longrightarrow} A \rightarrow 0 \\
\quad s^{\prime \prime} \Gamma^{3} A \downarrow \\
0 \longrightarrow S^{\prime} \Gamma A+P S A \stackrel{\phi^{\prime \prime}}{\longrightarrow} P S A+P S \Gamma A \stackrel{\psi^{\prime \prime}}{\longrightarrow} A^{\prime \prime} \rightarrow 0
\end{gathered}
$$

where

$$
\begin{aligned}
\phi & =u_{2} j_{1}^{\prime}\left(s^{\prime} \Gamma^{2} A\right) j_{2}+u_{2}^{\prime} j_{2}^{\prime}, \quad \psi=\left(s^{\prime \prime} \Gamma A\right)(p S \Gamma A) v_{2}, \\
\phi^{\prime \prime} & =u_{1} j^{\prime}\left(s^{\prime} \Gamma A\right) j_{1}+u_{1}^{\prime} j_{1}^{\prime}, \quad \psi^{\prime \prime}=-a^{\prime \prime}(p A) v_{1}, \\
\theta & =\left[u_{1}^{\prime}-u_{1} j^{\prime}(p S \Gamma A)\right] v_{2}+\left[u_{1} j^{\prime}\left(s^{\prime} \Gamma A\right) j_{1}+u_{1}^{\prime} j_{1}^{\prime}\right]\left(p S \Gamma^{2} A\right) v_{2}^{\prime} .
\end{aligned}
$$

The lower row is, except for the sign of $\psi^{\prime \prime}$, just the direct sum of the sequence $C$ of 4.2 with $0 \rightarrow P S A \rightarrow P S A \rightarrow 0 \rightarrow 0$ and is thus a proper s.e.s. The upper row plays a similar role with respect to $\Gamma^{2} A$. 
Moreover, the diagram commutes:

$$
\begin{aligned}
\psi^{\prime \prime} \theta & =a^{\prime \prime}(p A) j^{\prime}(p S \Gamma A) v_{2}-a^{\prime \prime}(p A) j^{\prime}\left(s^{\prime} \Gamma A\right)\left(p S \Gamma^{2} A\right) j_{1} v_{2}^{\prime} \\
& =a^{\prime \prime}\left(s^{\prime \prime} \Gamma A-a^{\prime} j\right)\left[(p S \Gamma A) v_{2}-\left(s^{\prime} \Gamma A\right) j_{1}\left(p S \Gamma^{2} A\right) v_{2}^{\prime}\right] \\
& =a^{\prime \prime}\left(s^{\prime \prime} \Gamma A\right)(p S \Gamma A) v_{2} \\
& =a^{\prime \prime} \psi
\end{aligned}
$$

while

$$
\begin{aligned}
\theta \phi= & {\left[u_{1}^{\prime}-u_{1} j^{\prime}(p S \Gamma A)\right] j_{1}^{\prime}\left(s^{\prime} \Gamma^{2} A\right) j_{2}+\left[u_{1} j^{\prime}\left(s^{\prime} \Gamma A\right) j_{1}+u_{1}^{\prime} j_{1}^{\prime}\right]\left(p S \Gamma^{2} A\right) j_{2}^{\prime} } \\
= & u_{1}^{\prime} j_{1}^{\prime}\left(s^{\prime} \Gamma^{2} A\right) j_{2}-u_{1} j^{\prime}\left[s^{\prime \prime} \Gamma^{2} A-\left(s^{\prime} \Gamma A\right) j_{1}\right]\left(s^{\prime} \Gamma^{2} A\right) j_{2} \\
& +u_{1} j^{\prime}\left(s^{\prime} \Gamma A\right) j_{1}\left(p S \Gamma^{2} A\right) j_{2}^{\prime}+u_{1}^{\prime} j_{1}^{\prime}\left(p S \Gamma^{2} A\right) j_{2}^{\prime} \\
= & u_{1} j_{1}^{\prime}\left(s^{\prime \prime} \Gamma^{3} A\right)+u_{1} j^{\prime}\left(s^{\prime} \Gamma A\right) j_{1}\left[\left(s^{\prime} \Gamma^{2} A\right) j_{2}+\left(p S \Gamma^{2} A\right) j_{2}^{\prime}\right] \\
= & \phi^{\prime \prime}\left(s^{\prime \prime} \Gamma^{3} A\right) .
\end{aligned}
$$

Now $s^{\prime \prime} \Gamma^{3} A$ and $a^{\prime \prime}$ are of course proper epimorphisms. But so also is $\theta$. For there is a map $w_{1}: P A \rightarrow P S T A$ such that $(p S \Gamma A) w_{1}=-i^{\prime}$, and a map $w_{2}: P S \Gamma A \rightarrow P S \Gamma^{2} A$ such that $\left(p S \Gamma^{2} A\right) w_{2}=i^{\prime}$. But then

$$
\begin{aligned}
& \theta\left(u_{2} w_{1} v_{1}+u_{2}^{\prime} w_{2} v_{1}^{\prime}\right) \\
& \quad=\left[u_{1}^{\prime}-u_{1} j^{\prime}(p S \Gamma A)\right] w_{1} v_{1}+\left[u_{1} j^{\prime}\left(s^{\prime} \Gamma A\right) j_{1}+u_{1}^{\prime} j_{1}^{\prime}\right]\left(p S \Gamma^{2} A\right) w_{2} v_{1}^{\prime} \\
& \quad=u_{1}^{\prime} w_{1} v_{1}+u_{1} v_{1}+u_{1}^{\prime} v_{1}^{\prime} \\
& \quad=1:(P S A+P S \Gamma A)+u_{1}^{\prime} w_{1} v_{1} .
\end{aligned}
$$

But this is an equivalence with inverse 1: $(P S A+P S \Gamma A)-u_{1}^{\prime} w_{1} v_{1}$, hence a proper epimorphism.

Thus the diagram above is part of the following diagram,

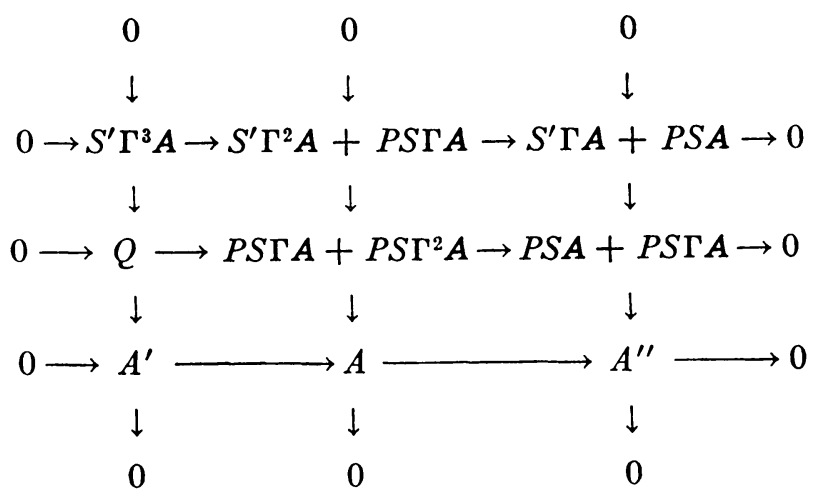

which commutes, and whose rows and columns are proper exact. The bottom row however is $A$, the top one is $\Gamma^{3} A$, and the middle one is projective in $\mathcal{K}^{8}$. Since (5.2) gives a perfectly legitimate assignment of projectives in $\mathfrak{K}^{s}$ it is possible to arrange that $\Gamma^{3}=\Omega$; in this case the canonical commutation 
equivalences are seen to be compositions of the canonical permutation equivalences, and of $\Omega, \Omega^{2}$ applied to these maps. In any case $\Gamma^{3}$ is equivalent to $\Omega$ on $K^{s}$, the equivalence giving rise, on each of the three terms, to maps derived from the commutation and permutation equivalences.

The canonical permutation and commutation equivalences, powers of $\Omega$ applied to these maps, and compositions of all these give a set of natural equivalences which will be referred to as the generalized commutation equivalences. The following proposition, which is essentially only an application of the above theorem and of Proposition 4.3, shows how some of them commute with certain other natural transformations.

Proposition 5.3. The diagrams

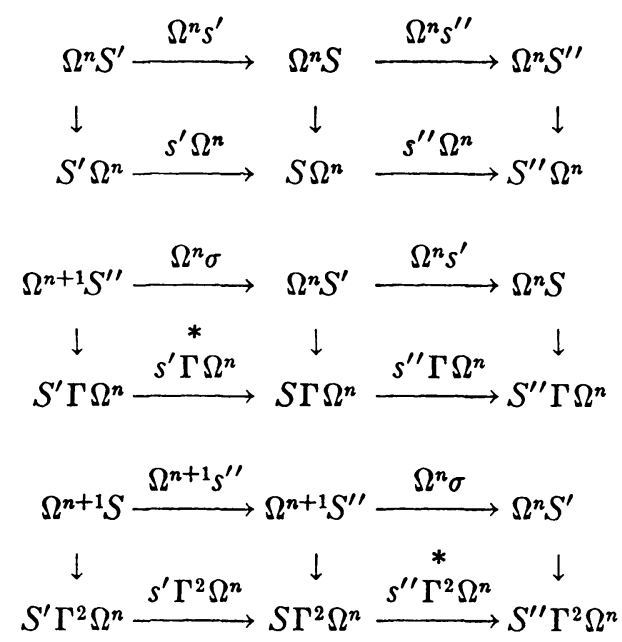

in which the vertical arrows represent generalized commutation equivalences, commute, in $\mathfrak{K} / \mathfrak{B}[\mathfrak{K}]$, in the unstarred squares and anticommute in the starred squares.

An immediate consequence of 5.3 is the following theorem.

THEOREM 5.4. If $\mathcal{K}$ has enough projectives, $B \in \Re$, and

$$
\boldsymbol{A}=\left(0 \rightarrow A^{\prime} \stackrel{a^{\prime}}{\rightarrow} \rightarrow A \stackrel{a^{\prime \prime}}{\rightarrow} \rightarrow A^{\prime \prime} \rightarrow 0\right)
$$

is a proper s.e.s. in $\nVdash$ then

$$
\begin{aligned}
& \cdots \rightarrow \operatorname{Hom}\left(B, \Omega^{n+1} A^{\prime \prime} ; \Re / \mathfrak{P}[\mathcal{K}]\right) \stackrel{\left(\Omega^{n} \sigma A\right) \circ}{\longrightarrow} \operatorname{Hom}\left(B, \Omega^{n} A^{\prime} ; \mathcal{K} / \mathfrak{P}[\mathcal{K}]\right) \\
& \stackrel{\left(\Omega^{n} a^{\prime}\right) \circ}{\longrightarrow} \operatorname{Hom}\left(B, \Omega^{n} A ; \Re / \mathfrak{P}[\mathcal{K}] \stackrel{\left(\Omega^{n} a^{\prime \prime}\right) \circ}{\longrightarrow} \operatorname{Hom}\left(B, \Omega^{n} A^{\prime \prime} ; \Re / \mathfrak{B}[\mathcal{K}]\right)\right. \\
& \rightarrow \cdots \rightarrow \operatorname{Hom}(B, A ; \Re / \mathfrak{P}[\Re]) \stackrel{a^{\prime \prime} \circ}{\longrightarrow} \operatorname{Hom}\left(B, A^{\prime \prime} ; \Re / \mathfrak{P}[\Re]\right)
\end{aligned}
$$

is exact. 
Properly, $\left(\Omega^{n} \sigma A\right) \circ$ should be written $\operatorname{Hom}\left(1: B, \Omega^{n} \sigma A ; \Re / \mathfrak{P}[\mathcal{K}]\right)$ and so forth.

In view of 5.3 it is enough to show that $\operatorname{Hom}\left(B, A^{\prime} ; \mathfrak{K} / \mathfrak{P}[\mathcal{K}]\right)$ $\rightarrow \operatorname{Hom}(B, A ; \Re / \mathfrak{P}[\mathfrak{K}]) \rightarrow \operatorname{Hom}\left(B, A^{\prime \prime} ; \mathfrak{K} / \mathfrak{P}[\mathfrak{K}]\right)$ is exact for any proper s.e.s. $A$, for 5.3 allows comparison, at any stage, with such an s.e.s.

The composition is obviously zero. If $f: B \rightarrow A, a^{\prime \prime} f \in \mathfrak{B}[\mathcal{K}]$ then $a^{\prime \prime} f=a^{\prime \prime} \phi$ for some $\phi \in \mathfrak{B}[\Re]$. But then $a^{\prime \prime}(f-\phi)=0$ and for $g: B \rightarrow A^{\prime}, a^{\prime} g=f-\phi$ $\equiv f \bmod \mathfrak{B}[\mathcal{K}]$ which completes the proof.

The same process can of course be applied to any half-exact functor which vanishes on $\mathfrak{B}[\mathcal{K}]$.

The duals of the above results are also true. If in particular the category is a Frobenius category then the sequence of 5.4 is defined, and exact, for negative as well as positive $n$.

6. Comparison with Ext; the category $\varepsilon \mathcal{K}$. If $\mathscr{K}$ is an abelian category with enough projectives the graded additive category $\mathcal{E} \mathscr{K}$ has as objects just the objects of $\mathscr{K}$; if $A, A^{\prime} \in \mathcal{E} \mathcal{K}$ then

$$
\operatorname{Hom}_{-q}\left(A, A^{\prime} ; \varepsilon \Re\right)=\operatorname{Hom}\left(\Omega^{q} A, A^{\prime} ; \Re / \mathfrak{B}[\Re]\right) \text { for } q \geqq 0 \text {, }
$$

the groups being 0 in positive degrees; if $f: \Omega^{a} A \rightarrow A^{\prime}, g: \Omega^{r} A^{\prime} \rightarrow A^{\prime \prime}$ in $\mathscr{K} / \mathfrak{B}[\mathcal{K}$ ] then the composition $g \circ f$ in $\mathcal{E} K$ is $(-1)^{a r} g\left(\Omega^{r} f\right)$, computed in $\mathscr{K} / \mathfrak{P}[\mathcal{K}]$. The proof that this defines an additive category is straightforward and will be omitted. Notice that $\mathscr{K} / \mathfrak{P}[\mathcal{K}]$ is just the subcategory of $\mathcal{E} K$ consisting of maps of degree 0 .

$\varepsilon \mathcal{K}$ is in fact just a quotient of the extended category Ext $\mathcal{K}$ defined in [AC, §12]. This relation is exhibited by a functor $E$ : Ext $\mathcal{K} \rightarrow \mathcal{E} \nVdash$ defined as follows. $E$ is the identity on objects. On maps of degree 0 it is just the identification functor $\mathfrak{K} \rightarrow \mathfrak{K} / \mathfrak{P}[\mathcal{K}]$. For $q>0$ an element of $\operatorname{Hom}_{-q}\left(A, A^{\prime}\right.$; Ext $\left.\mathfrak{K}\right)$ is a homotopy class of maps $f: P R A \rightarrow P R A^{\prime}$ of degree $-q$. For such a map consider

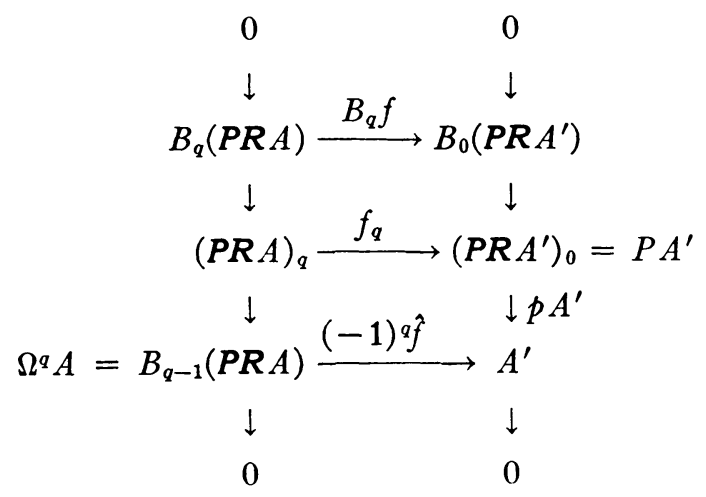

where the columns are proper s.e.s. and the upper square commutes; $\hat{f}$ is 
defined to make the lower square commute. If $f=\partial g \pm g \partial$ then $\left(p A^{\prime}\right) f_{q}$ $= \pm\left(p A^{\prime}\right) g \beta \delta$ so that $\hat{f}= \pm g \beta \in \mathfrak{B}[\mathcal{K}]$. Thus $f \rightarrow \hat{f}$ defines a homomorphism $E: \operatorname{Hom}\left(A, A^{\prime} ; \operatorname{Ext} \mathcal{K}\right) \rightarrow \operatorname{Hom}\left(A, A^{\prime} ; \mathcal{E} \mathcal{K}\right)$; it remains to be proved that $E$ is a functor.

THEOREM 6.2. E: Ext $\Re \rightarrow \mathcal{K} K$ is a functor; in addition for any $A, A^{\prime} \in \mathcal{K}$, $E: \operatorname{Hom}\left(A, A^{\prime} ; \operatorname{Ext} \mathfrak{K}\right) \rightarrow \operatorname{Hom}\left(A, A^{\prime} ; \mathcal{E} \mathcal{K}\right)$ is an epimorphism. Finally, the kernel of $E$ is generated by the projectives of $\varkappa$.

For the first statement it is necessary to prove only that $E$ preserves compositions; this needs discussion only when both maps are of strictly negative degree. Suppose $f$ is as above and $g: P R A^{\prime} \rightarrow P R A^{\prime \prime}$ is of degree $-r$ for $r>0$. Consider the diagram

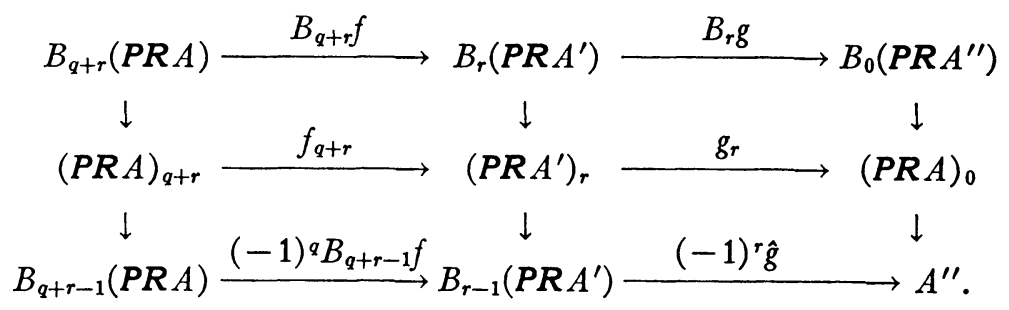

This commutes, $g_{r} f_{q+r}=(g f)_{q+r}$ and $\left(B_{r} g\right)\left(B_{q+r} f\right)=B_{q+r}(g f)$. Thus $\widehat{g f}$ $=\hat{\mathrm{g}} B_{q+r-1} f$. Now 6.1 shows that $B_{q} f=(-1)^{q} \Omega \hat{f}$ in $\mathcal{K} / \mathfrak{B}[\mathcal{K}]$; induction on $r$ (see the left side of 6.3 ) shows that $B_{q+r-1} f=(-1)^{{ }^{a r}} \Omega^{r} \hat{f}$.

The second statement is trivial; in fact for any $\phi: B_{q-1}(P R A) \rightarrow A^{\prime}$ it is easy to produce an $f$ with $\hat{f}=\phi$.

For the third observe that in (6.1), $\left(p A^{\prime}\right) f_{q}= \pm \hat{f} \delta \in Z^{q}\left(P R A ; A^{\prime}\right)$ is a coboundary if and only if $f$ is nullhomotopic, i.e. if $\hat{f}=\psi \beta$ for some $\psi:(P R A)_{q-1}$ $\rightarrow A^{\prime}$. A fortiori if $\hat{f}=0$ then $f$ is nullhomotopic.

Now suppose $\hat{f} \in \mathfrak{B}[\varkappa]$. Then $\hat{f}=\left(p A^{\prime}\right) \phi$ for some $\phi: B_{q-1}(P R A) \rightarrow P A^{\prime}$. But then $\phi \delta: P R A \rightarrow P A^{\prime}$ in $\partial \nVdash^{\infty}$. If $i: P A^{\prime} \rightarrow P R A^{\prime}$ is the injection, also a map in $\partial K^{\infty}$, then $(f-i \phi \delta)^{\wedge}=0$. Thus $f$ is homotopic to $i \phi \delta$ and its homotopy class is factored by elements of the groups $\operatorname{Hom}_{-q}\left(A, P A^{\prime}\right.$; Ext $\left.\mathscr{K}\right)$, $\mathrm{Hom}_{0}\left(P A^{\prime}, A^{\prime}\right.$; Ext $\left.\Re\right)$.

This result is complemented by the following proposition.

Proposition 6.4. If

$$
A=\left(0 \rightarrow A^{\prime} \stackrel{a^{\prime}}{\rightarrow} A \stackrel{a^{\prime \prime}}{\rightarrow} A^{\prime \prime} \rightarrow 0\right)
$$

is a proper s.e.s. in $\mathfrak{K}$ then $E(\Delta A)=\sigma A$.

To compute $\Delta A, A$ may be resolved in $\mathfrak{K}^{8}$ so that the resolution is a proper s.e.s.

$$
0 \rightarrow P R A^{\prime} \stackrel{\mathbf{a}^{\prime}}{\rightarrow} P R A \stackrel{\mathbf{a}^{\prime \prime}}{\rightarrow} P R A^{\prime \prime} \rightarrow 0
$$


in $\partial K^{\infty}$. If $\gamma: P R A^{\prime \prime} \rightarrow P R A$ splits this in $K^{\infty}$ and $a^{\prime} f=\partial \gamma-\gamma \partial$ then $f$ belongs to $\Delta \boldsymbol{A}$. Now consider the diagram

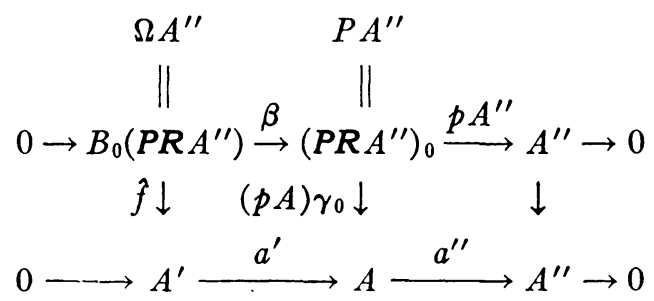

in $\mathcal{K}$. This commutes: $a^{\prime \prime}(p A) \gamma_{0}=\left(p A^{\prime \prime}\right) \mathbf{a}_{0}^{\prime \prime} \gamma_{0}=p A^{\prime \prime}$, while $a^{\prime} \hat{f} \delta=-a^{\prime}\left(p A^{\prime}\right) f_{1}$ $=-(p A) a_{0}^{\prime} f_{1}=-(p A)\left(\partial \gamma_{1}-\gamma_{0} \partial\right)=(p A) \gamma_{0} \beta \delta$ so that $a^{\prime} \hat{f}=(p A) \gamma_{0} \beta$. Thus $\hat{f}$ is in fact in $\sigma A$.

The following remarks on the consequences of these theorems will conclude this paper. If $F: \mathfrak{K} \rightarrow \mathscr{L}$ is a proper covariant additive functor then the left-derived functor $L F:$ Ext $\mathfrak{K} \rightarrow \mathscr{L}^{\infty}$ is defined [AC, $\left.\S 13\right]$. If only positive degrees are considered, i.e. $L F$ is altered to $L^{+} F$ by defining $L_{q}^{+} F=L_{q} F$, $q>0, L_{0}^{+} F=0$, then $L^{+} F$ clearly vanishes on the projectives of $\mathscr{K}$ and is thus a functor on $\varepsilon \mathcal{K}$. By 6.4 , its connecting transformation is $\left(L^{+} F\right) \sigma$. Similar remarks may be made for contravariant functors.

The kernel of $E$, in strictly negative degrees, is generated by the groups $\operatorname{Hom}_{-q}(A, X$; Ext $\mathfrak{K})$ with $X$ projective. It thus measures the failure of projectives to be injective. In a self-injective category, i.e. one in which all projectives are injective, the kernel of $E$ is just $\mathfrak{B}[\Re]$ so that for $q>0$, $E: \operatorname{Hom}_{-q}\left(A, A^{\prime} ;\right.$ Ext $\left.\mathfrak{K}\right) \approx \operatorname{Hom}_{-q}\left(A, A^{\prime} ; \varepsilon \mathscr{K}\right)$. In virtue of $6.4, E$ takes the exact sequences for Ext, [AC, 12.1], into exact sequences of homomorphism groups in $\mathcal{E}$, at least for degrees different from 0 .

In particular this is true in Frobenius categories, where it seems natural to extend the category $\mathcal{E} K$ to $\mathcal{E}^{\cdot} \mathcal{K}$ by defining $\operatorname{Hom}_{-q}\left(A, A^{\prime} ; \mathcal{E}^{\cdot} \mathcal{K}\right)$ $\operatorname{Hom}\left(\Omega^{a} A, A^{\prime} ; \mathfrak{K} / \mathfrak{B}[\mathcal{K}]\right)$ for $q$ negative as well as positive. The exact sequences for Ext are then embedded, except for terms of degree 0 , in sequences having in general nontrivial terms of all degrees.

It should, finally, be observed that all the above remarks are subject to dualization, which is not here made explicit.

\section{BIBLIOGRAPHY}

[AC] Alex Heller, Homological algebra in abelian categories, Ann. of Math. vol. 68 (1958) pp. 484-525.

[G] A. Grothendieck, Sur quelques points d'algebre homologique, Tôhoku Math. J. vol. 9 (1957) pp. 119-221.

[HE] P. J. Hilton, Homotopy theory of modules and duality, Symposium Internacional de Topologia Algebraica, Mexico, 1958 pp. 273-281.

UNIVERSITY OF ILLINOIS,

URBANA, ILLINOIS 